\title{
Bidirectional Carouseling Method Based on Phase Difference for MEMS Gyro North Finder
}

\author{
Xiaowen Cai, Chunxi Zhang, Shuang Gao, ${ }^{*}$ and Ruoyu Zhang \\ School of Instrumentation Science and Opto-electronics Engineering, \\ Beihang University, Beijing 100191, China
}

(Received March 7, 2019; accepted July 16, 2019)

Keywords: MEMS gyro, north finder, bias drift, bidirectional, phase difference

The north finder has been widely used in precision guided weapons, unmanned aerial vehicles (UAVs), and other device. In this paper, a north finder based on a low-cost microelectro-mechanical system (MEMS) gyro is proposed. In order to reduce the substantial bias effect on the gyro's north finding angle, a bidirectional carouseling method is applied. To improve the north finding accuracy, the phase difference between the turntable rotation rate and the gyro output is used. After the calculation of the phase difference, a five-order Butterworth filter is chosen to eliminate the gyro high-frequency noise. Experimental results demonstrate that the proposed method based on the phase difference can effectively improve the north finding accuracy by $50 \%$ compared with the traditional carouseling method.

\section{Introduction}

The north finder is widely used in military and civilian fields such as targeting, dead reckoning, pointing system, and inertial navigation. Traditional gyro north finders commonly use high-precision gyros, such as fiber optic gyros (FOGs), ring laser gyros (RLGs), and hemispherical resonator gyros (HRGs). However, the high-precision gyros are bulky and expensive. ${ }^{(1)}$ Their large volume, high power consumption, and high cost limit their applications in portable navigation. With the rapid development of the micro-electromechanical system (MEMS) technique, the precision and stability of MEMS gyros have been significantly improved in recent years. ${ }^{(2)}$ Owing to its small size, low cost, low power, and environment adaptability, ${ }^{(3,4)}$ a MEMS gyro north finder has become a research focus in the north finder field. However, limited by its mechanical precision, a MEMS gyro is one or two orders of magnitude worse than a high-precision gyro; ${ }^{(5)}$ thus, effective methods must be developed to improve its measurement accuracy. In order to realize a MEMS gyro north finder, there are two main traditional methods: a static method (maytagging) and a dynamic method (carouseling).

Maytagging is carried out by discrete $\pm 180^{\circ}$ turning of the gyro's sensitive axis, and it allows a gyro to face in a given direction while collecting data at the desired interval. Maytagging has been widely applied in various methods of north finding angle detection, such as the two-

*Corresponding author: e-mail: gaoshuang@buaa.edu.cn https://doi.org/10.18494/SAM.2019.2361 
point method, four-point method, and multiposition method. ${ }^{(6-8)}$ It is simple to operate and is widely employed in practical applications. However, this method will increase the error caused by the rate random walk (RRW), which conflicts with the angular random walk (ARW). In contrast to maytagging, carouseling has great advantages in terms of the robustness against bias drift, ${ }^{(9)}$ angular random walk, and temperature drift. The north finding angle is measured by the turntable continuous rotation. However, the angular position error of the turntable will bring additional uncertainties to the accuracy of north finding in this method. Draper Laboratory has been developing a MEMS north finder for over a decade and has become a world leader in this field. ${ }^{(10)}$ Developers from Honeywell Laboratory have reported a process for a MEMS tuning-fork-type gyro with the azimuth angle maintained in $30 \mathrm{~s},{ }^{(1)}$ and long-term bias drift was reduced by carouseling. Trusov and coworkers discussed how carouseling can reduce the north finding time and decrease the error caused by bias drift and angular random walk. ${ }^{(12,13)}$ Renkoski showed progress in the development of high-accuracy MEMS gyros with north-finding capabilities, so that the errors can be eliminated by an improved carouseling method called the baseball stitch slew. ${ }^{(10)}$ Prikhodko et al. obtained the north finding angle by detecting the difference between the phase of a sinusoidal fit and the instantaneous position of the turntable. ${ }^{(14)}$ The uncertainty was reduced by data averaging and the robustness against bias drift in carouseling was proved evidently. However, few applications of carouseling have been realized in the industrial field; thus, there is a great potential to develop a novel method to improve carouseling and put it into practical use.

In order to overcome the shortcomings of traditional methods, in this paper we present a novel carouseling method. The proposed method aims to achieve the following functions:

(1) An improved carouseling method is proposed to decrease MEMS gyro drift;

(2) The phase difference between the turntable position signal and MEMS gyro output is utilized to further reduce the gyro drift and improve north finding accuracy;

(3) A five-order Butterworth filter is used to eliminate the gyro high-frequency noise.

The remainder of this work is organized as follows. In Sect. 2, the theoretical basis of the methodology of MEMS gyro north finder is presented. The implementation of the proposed system is introduced in detail in Sect. 3. The experimental results in Sect. 4 validate the feasibility of the proposed method. To sum up, conclusions are drawn in Sect. 5.

\section{Methodology of North Finder}

At a certain local terrestrial latitude $L$, the rotation rate is divided into two parts, the vertical component and the horizontal component, ${ }^{(15)}$ as shown in Fig. 1. The gyro's reference coordinate system is coincident with the local geographic coordinate system $X_{g}, Y_{g}$, and $Z_{g}$, denoting the geographical east, north, and up directions, respectively. The MEMS gyro is fixed perpendicular to the horizontal plane and the sensitive axis is paralleled to the horizontal plane. ${ }^{(16,17)}$ $\alpha$ in the gyro's coordinate system in the $\mathrm{O}-X_{g} Y_{g}$ plane is the north finding angle.

As shown in Fig. 1, $\omega_{e}$ is Earth's rotation rate, which is $15.041 \% \mathrm{~h}^{(18)}$ The horizontal component of Earth's rotation rate is described as

$$
\omega_{N}=\omega_{e} \cos L .
$$




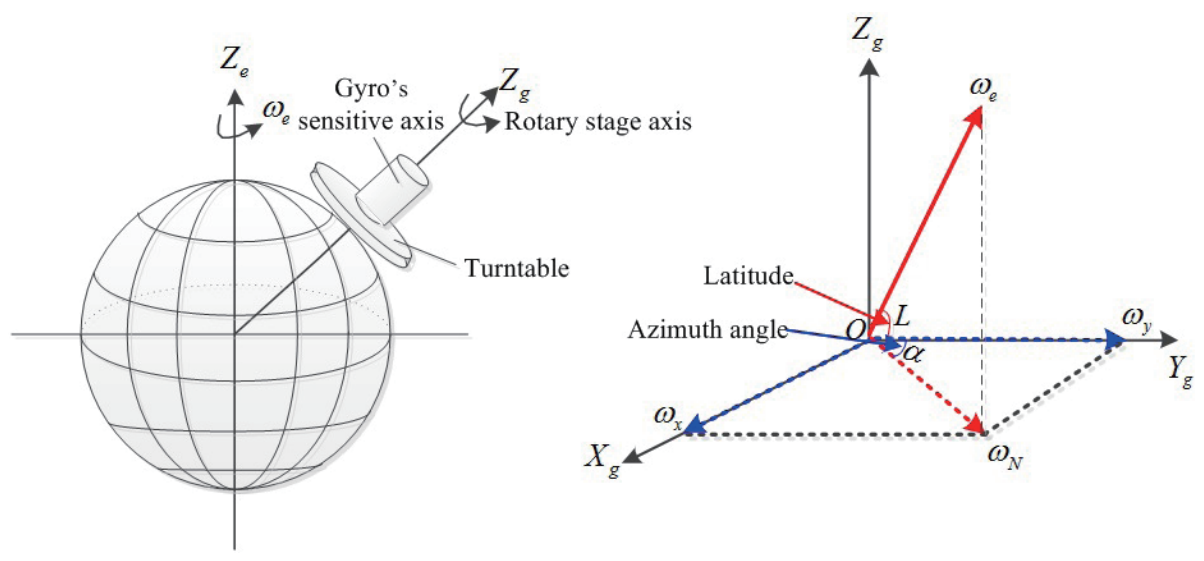

Fig. 1. (Color online) Geographic coordinate system on the gyro's location.

Assuming that $Y_{g}$ represents the true north direction, the north component of Earth's rotation rate is

$$
\omega_{y}=\omega_{N} \cos \alpha .
$$

According to Eq. (2), the north finding angle $\alpha$ can be obtained by measuring the angular rate of the gyro.

\section{Carouseling Method for North Finder}

\subsection{Traditional carouseling for north finder}

The traditional carouseling method measures Earth's rotation rate on the basis of the continuous rotation of the MEMS gyro. As shown in Fig. 2, when the orientation of the gyro's sensitive axis is rotated continuously, the gyro output appears to be a sinusoidal signal with the variation of the angle between Earth's rotation axis and the gyro's sensitive axis.

According to the mathematical model, when the turntable is rotated at an angular rate $\omega_{t}$, the dynamic output model of the MEMS gyro's at the north finding angle $\alpha$ in the horizontal plane can be expressed as

$$
\omega(t)=K \omega_{N} \cos \left(\omega_{t} t+\alpha\right)+b_{c}+\varepsilon(t),
$$

where $\omega(t)$ is the gyro dynamic output, $K$ is the gyro scale factor, $\omega_{N}$ is the vertical component of Earth's rotation rate, $\varepsilon(t)$ is the gyro random drift, and $b_{c}$ is the gyro constant bias. The gyro output can be discretized as 


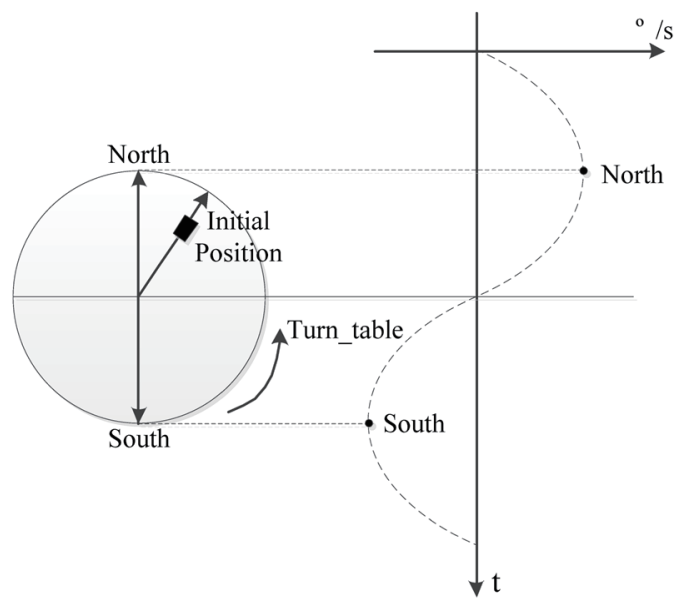

Fig. 2. Curve of Earth and turntable combined action.

$$
\omega\left(i / f_{s}\right)=K \omega_{N} \cos \left[2 \pi\left(f_{t} / f_{s}\right) i+\alpha\right]+b_{c}+\varepsilon\left(i / f_{s}\right), \quad(i=1,2, \cdots, N)
$$

where $f_{t}$ is the turntable frequency, $f_{s}$ is the sampling frequency, and $i$ is the sampling point number. According to Eq. (4), Eq. (5) can be obtained by matrix decomposition.

$$
\begin{aligned}
\omega\left(i / f_{s}\right)= & K \omega_{N} \cos \left[2 \pi\left(f_{t} / f_{s}\right) i+\alpha\right]+b_{c}+\varepsilon\left(i / f_{s}\right) \\
& =K \omega_{N}\left\{\cos \left[2 \pi\left(f_{t} / f_{s}\right) i\right] \cos \alpha-\sin \left[2 \pi\left(f_{t} / f_{s}\right) i\right] \sin \alpha\right\} \\
& +b_{c}+\varepsilon\left(i / f_{s}\right) \\
& {\left[\begin{array}{c}
K \omega_{N} \cos \left[2 \pi\left(f_{t} / f_{s}\right) i\right] \\
-K \omega_{N} \sin \left[2 \pi\left(f_{t} / f_{s}\right) i\right] \\
1 \\
\varepsilon\left(i / f_{s}\right)
\end{array}\right]^{\mathrm{T}}\left[\begin{array}{c}
\cos \alpha \\
\sin \alpha \\
b_{c} \\
1
\end{array}\right] \quad(i=1,2, \cdots, N) }
\end{aligned}
$$

The least squares method (LSM) ${ }^{(19)}$ can be used to solve the problem. Denote

$$
\begin{aligned}
& Y=\omega\left(i / f_{s}\right)=\left[\omega\left(1 / f_{s}\right), \omega\left(2 / f_{s}\right), \cdots, \omega\left(N / f_{s}\right)\right], \\
& X=\left[\begin{array}{lll}
\cos \alpha \sin \alpha & b_{c} & 1
\end{array}\right]^{\mathrm{T}} \text {, and } \\
& A=\left[\begin{array}{cccc}
K \omega_{N} \cos \left[2 \pi\left(f_{t} / f_{s}\right)\right] & -K \omega_{N} \sin \left[2 \pi\left(f_{t} / f_{s}\right)\right] & 1 & \varepsilon\left(1 / f_{s}\right) \\
K \omega_{N} \cos \left[2 \pi\left(2 f_{t} / f_{s}\right)\right] & -K \omega_{N} \sin \left[2 \pi\left(2 f_{t} / f_{s}\right)\right] & 1 & \varepsilon\left(2 / f_{s}\right) \\
\vdots & \vdots & \vdots & \vdots \\
K \omega_{N} \cos \left[2 \pi\left(N f_{t} / f_{s}\right)\right] & -K \omega_{N} \sin \left[2 \pi\left(N f_{t} / f_{s}\right)\right] & 1 & \varepsilon\left(N / f_{s}\right)
\end{array}\right] .
\end{aligned}
$$


Equation (5) can be written as

$$
Y=A X .
$$

Equation (7) can be reckoned by LSM solutions. Then, the north finding angle $\alpha$ can be calculated as

$$
\begin{gathered}
\hat{X}=\left(A^{\mathrm{T}} A\right)^{-1} A^{\mathrm{T}} Y, \\
\alpha=\arctan \left(\frac{A \sin \alpha}{A \cos \alpha}\right)=\arctan \left(\frac{X_{11}}{X_{12}}\right) .
\end{gathered}
$$

\subsection{Bidirectional carouseling method based on phase difference}

The traditional carouseling method has the advantages of flexibility in designing parameters, real-time north finding, and simple operation. Under the same conditions, such as the north finding time or the number of samples collected, the north finding error of the carouseling method is much larger than that of the maytagging method. Thus, the north finding performance of the carouseling method is not ideal for practical use. In order to reduce the effect of bias drift on north finding accuracy, the mechanism of gyro drift is analyzed and a bidirectional carouseling method based on the phase difference is proposed.

\subsubsection{Effect of MEMS gyro output and rotation rate instability error of the turntable}

According to Eq. (3), by ignoring the mechanical vibration of the turntable and considering the gyro drift and the rotation rate instability error of the turntable, we obtain the actual gyro output as

$$
\begin{aligned}
\omega(t) & =K \omega_{N} \cos \left[\left(\omega_{t}+\Delta \omega_{t}\right) t+\alpha\right]+b_{c}+\varepsilon(t) \\
& =K \omega_{N} \cos \left[\left(\omega_{t}+\Delta \omega_{t}\right) t\right] \cos \alpha-\sin \left[\left(\omega_{t}+\Delta \omega_{t}\right) t\right] \sin \alpha+b_{c}+\varepsilon(t),
\end{aligned}
$$

where $\Delta \omega_{t}$ is the rotation rate instability error of the turntable and $\varepsilon(t)$ is a slowly time-varying random signal, which is an important performance parameter of the MEMS gyro and also the important component affecting north finding accuracy. To reduce the impact of $\varepsilon(t)$, there are two methods to solve the problem: the appropriate north finding solution method and the digital filtering method. In this paper, we choose a bidirectional carouseling method to solve the problem.

Equation (10) shows that the north finding angle error is directly caused by the rotation rate instability error of the turntable. At present, the technology for stable-speed servos is quite mature. The turntable used in this paper is a high precision turntable, the rotation rate instability error is small and can be neglected in general. 


\subsubsection{Bidirectional carouseling method based on phase difference}

The position signal of the turntable is an angle value, which increases from an initial position to $360^{\circ}$. The position signal of the turntable can be assumed as a periodic signal; then, the position signal $P_{t}(t)$ can be described as

$$
P_{t}(t)=\cos \left(\omega_{t} t\right)
$$

where $P_{t}(t)$ has no unit because it represents the value on the unit circle.

Assume that the phase difference between the gyro output and the turntable position signal is the north finding angle $\alpha$. The curves of the MEMS gyro output $\omega(t)$ and the position signal $P_{t}(t)$ are shown in Fig. 3.

When the turntable rotation mode is bidirectional, Eqs. (12) and (13) can be substituted for Eq. (11) as

$$
\begin{gathered}
P_{t_{-} C W}(t)=\cos \left(\omega_{t_{-} C W} t\right), \\
P_{t_{-} C C W}(t)=\cos \left(\omega_{t_{-} C C W} t\right),
\end{gathered}
$$

where $\omega_{t_{-}} W_{t} t$ is the gyro clockwise output and $\omega_{t_{-} C C W t}$ is the gyro counterclockwise output. By multiplying the position signal of the turntable and MEMS gyro output, we describe the differential phase $N(t)$ as

$$
N(t)=P_{t}(t) \omega(t)
$$

By using Eqs. (3), (12), and (13), we obtain the differential phases $N_{C W}(t)$ and $N_{C C W}(t)$ through bidirectional rotation as

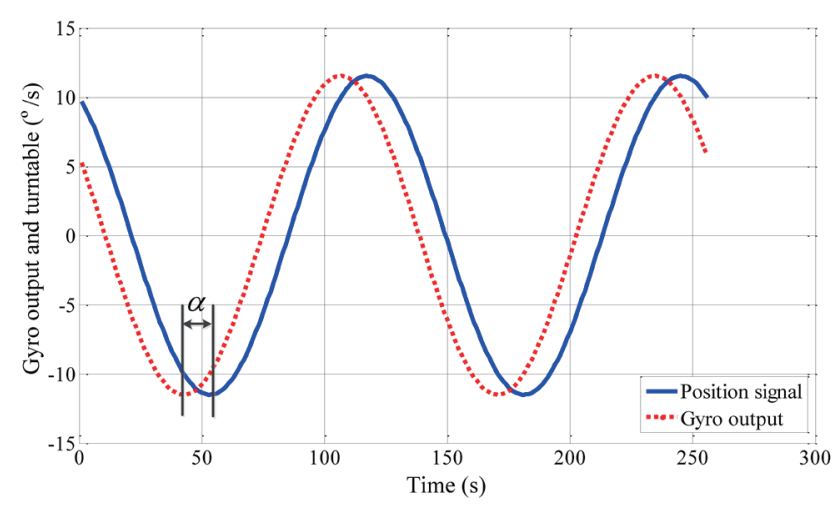

Fig. 3. (Color online) Curves of MEMS gyro output and the position signal. 


$$
\begin{aligned}
N_{C W}(t)= & P_{t_{-} C W}(t) \omega_{C W}(t) \\
= & \cos \left(\omega_{t_{-} C W} t\right)\left[K \omega_{N} \cos \left(\omega_{t_{-} C W} t+\alpha\right)+b_{c_{-} C W}+\varepsilon_{C W}(t)\right] \\
= & \cos \left(\omega_{t_{-} C W} t\right) K \omega_{N} \cos \left(\omega_{t_{-} C W} t+\alpha\right) \\
& +\cos \left(\omega_{t_{-} C W} t\right) b_{c_{-} C W}+\cos \left(\omega_{t_{-} C W} t\right) \varepsilon_{C W}(t) \\
= & \frac{1}{2} \omega_{N} \cos \left(2 \omega_{t_{-} C W} t+\alpha\right)+\frac{1}{2} \omega_{N} \cos \alpha \\
& +\cos \left(\omega_{t_{-} C W} t\right)\left(b_{c_{-} C W}+\varepsilon_{C W}(t)\right), \\
N_{C C W}(t)= & P_{t_{-} C C W}(t) \omega_{C C W}(t) \\
= & \cos \left(\omega_{t_{-} C C W} t\right)\left[K \omega_{N} \cos \left(\omega_{t_{-} C C W} t+\alpha\right)+b_{c_{-} C C W}+\varepsilon_{C C W}(t)\right] \\
= & \cos \left(\omega_{t_{-} C C W} t\right) K \omega_{N} \cos \left(\omega_{t_{-} C C W} t+\alpha\right) \\
& +\cos \left(\omega_{t_{-} C C W} t\right) b_{c_{-} C C W}+\cos \left(\omega_{t_{-} C C W} t\right) \varepsilon_{C C W}(t) \\
= & \frac{1}{2} \omega_{N} \cos \left(2 \omega_{t_{-} C C W} t+\alpha\right)+\frac{1}{2} \omega_{N} \cos \alpha \\
& +\cos \left(\omega_{t_{-} C C W} t\right)\left(b_{c_{-} C C W}+\varepsilon_{C C W}(t)\right),
\end{aligned}
$$

where $N_{C W}(t)$ and $N_{C C W}(t)$ represent the differential phases of clockwise and counterclockwise rotations, respectively. $P_{t_{-} C W}(t)$ and $P_{t_{-} C C W}(t)$ are the bidirectional rotation of the turntable position signals.

On the basis of the phase difference, the constant and random drifts of MEMS gyro are modulated into periodic signals using a low-pass filter. Considering the calculation complexity and filtering performance, a five-order Butterworth filter is chosen to eliminate the periodic signal. $^{(20)}$ Through the Butterworth filter, all periodic signals in Eqs. (15) and (16) are filtered out, leaving the second term with the phase difference. After the modulation using the low-pass filter, Eqs. (15) and (16) can be simplified as

$$
\begin{gathered}
N_{C W}(t)=P_{t_{-} C W}(t) \omega_{C W}(t)=\frac{1}{2} \omega_{N} \cos \alpha, \\
N_{C C W}(t)=P_{t_{-} C C W}(t) \omega_{C C W}(t)=\frac{1}{2} \omega_{N} \cos \alpha .
\end{gathered}
$$

By adding Eqs. (17) and (18), we can calculate the north finding angle as

$$
\begin{gathered}
N_{C W}(t)+N_{C C W}(t)=\omega_{N} \cos \alpha, \\
\alpha=\arccos \frac{N_{C W}(t)+N_{C C W}(t)}{\omega_{N}} .
\end{gathered}
$$


According to the derivation process, the signal modulation based on the phase difference between the position signal of the turntable and the MEMS gyro output in bidirectional rotation eliminates the effects of gyro drift and is not affected by the turntable accuracy.

\section{Experiments and discussion}

\subsection{North finder scheme and MEMS gyro characteristics}

On the basis of the aforementioned north finder that was previously described, the proposed hardware design is shown in Fig. 4. The MEMS gyro used was ADIS16495, which is produced by Analog Devices, Inc. ${ }^{(21)}$ MEMS gyro characteristics are shown in Table 1. The experiments were conducted at room temperature in Beijing, China, at latitude $39.97^{\circ}$ and longitude $116.34^{\circ}$.

The Allan variance was used to analyze the characteristics of the MEMS gyro. To plot the Allan deviation, data of the MEMS gyro were collected over $55 \mathrm{~h}$. The Allan deviation is shown in Fig. 5. As shown in Fig. 5, gyro noise contributes to the Allan deviation. There is
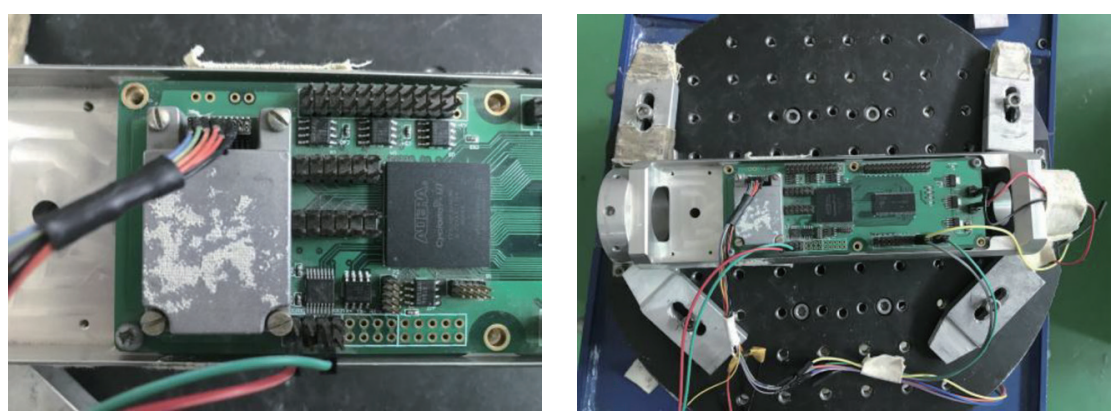

Fig. 4. (Color online) The proposed MEMS north finder.

Table 1

MEMS gyro characteristics.

\begin{tabular}{lccc}
\hline Parameter & Typical value & Parameter & Typical value \\
\hline Gyro range & $\pm 125^{\circ} / \mathrm{s}$ & In-Run Bias Stability & $0.8^{\circ} / \mathrm{h}$ \\
Nonlinearity & $\pm 0.2 \%$ of FS & Rate Noise Density & $0.002^{\circ} / \mathrm{s} / \sqrt{\mathrm{Hz}}(\mathrm{RMS})$ \\
Misalignment & $\pm 0.005^{\circ}$ & Angle Random Walk & $0.56^{\circ} / \sqrt{\mathrm{h}}$ \\
Repeatability & $\pm 0.07^{\circ} \mathrm{s}$ & Error Over Temperature & $\pm 0.2 \%$ \\
\hline
\end{tabular}

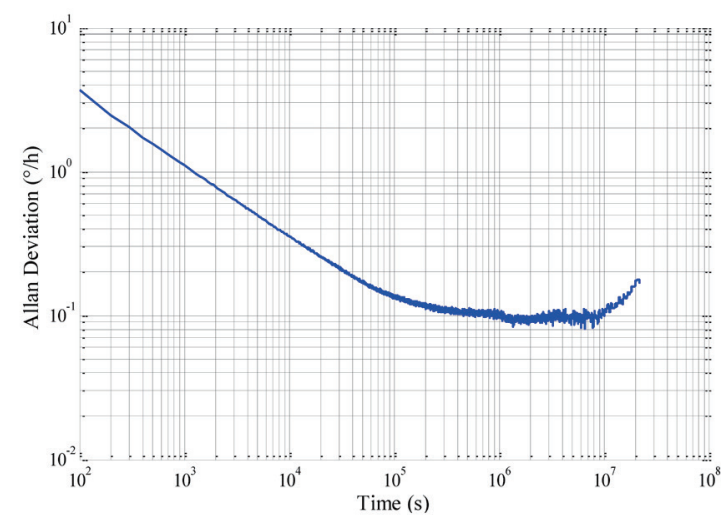

Fig. 5. (Color online) MEMS gyro Allan deviation. 
a direct relationship between the standard deviation of the output vs time and the slope of the Allan deviation. From Fig. 5, we can determine that the gyro ARW is $0.54 \% \sqrt{\mathrm{h}}$ and the gyro instability is $0.14 \% \mathrm{~h}$.

\subsection{Experiment on phase difference}

Because the low-pass filter was used, a higher rotation rate was found to be suitable for the experiment. The initial position was set to $0^{\circ}$, the designed rotation rate was $\pm 100 \%$, the sampling frequency was $10 \mathrm{~Hz}$, and output data for 100 cycles were collected during a period of 360 s. The MEMS gyro original output data are shown in Fig. 6.

As shown in Fig. 6, the gyro output signal contains a large number of different frequencies of gyro drift. The gyro drift at different frequencies was analyzed on the basis of power spectral densities (PSD) shown in Fig. 7. In order to reduce the noise and improve the signalto-noise ratio, the gyro output requires a suitable low-pass filter to deal with the filtered gyro output. A five-order Butterworth filter was chosen to filter the gyro output. ${ }^{(22)}$ The frequency response of the Butterworth filter was maximally flat in the passband and rolled off to zero in the stopband. This characteristic is suitable for the gyro in this experiment. The filtered results

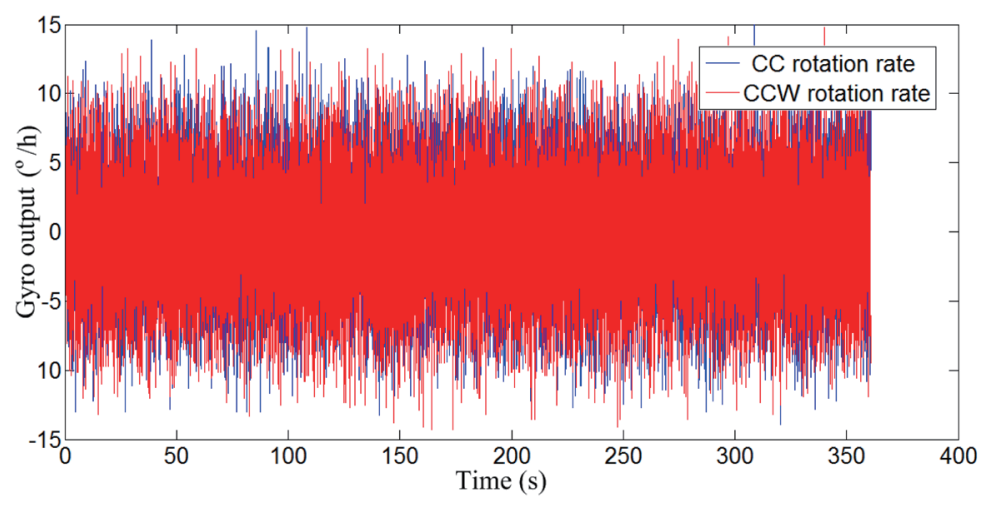

Fig. 6. (Color online) MEMS gyro original output.

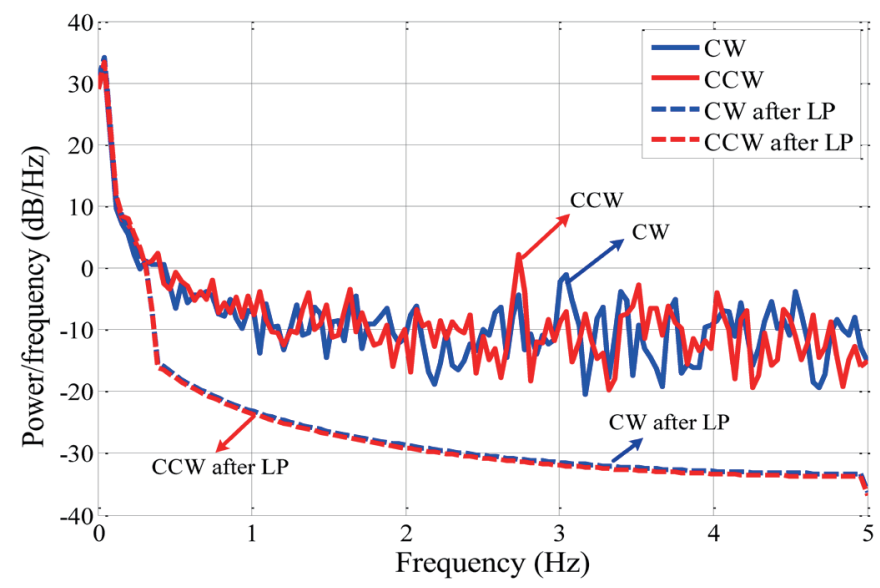

Fig. 7. (Color online) PSD and low-pass filter results. 
are also shown in Fig. 7. The result demonstrates that the high-frequency component has almost been eliminated apparently, but the low-frequency component still remains.

\subsection{Results of carouseling method at different rotation rates}

Assuming that the initial position of the MEMS gyro is coincident with the true north, the turntable rotation rate increases from $1 \%$ to $100 \%$ at $5 \%$ s-rate intervals, and the sample frequency is $10 \mathrm{~Hz}$. When the turntable rotation is bidirectional, the gyro outputs are collected on the basis of three typical rotation rates of the turntable, as shown in Fig. 8.

In order to compare the proposed method and the traditional method, the north finding angle was also obtained by the traditional carouseling method. The gyro output data for 20 cycles were collected and the relationship between the result of north finding and the turntable rate is shown in Fig. 9. The 1-sigma error bars of the proposed and traditional methods are shown in Fig. 10.

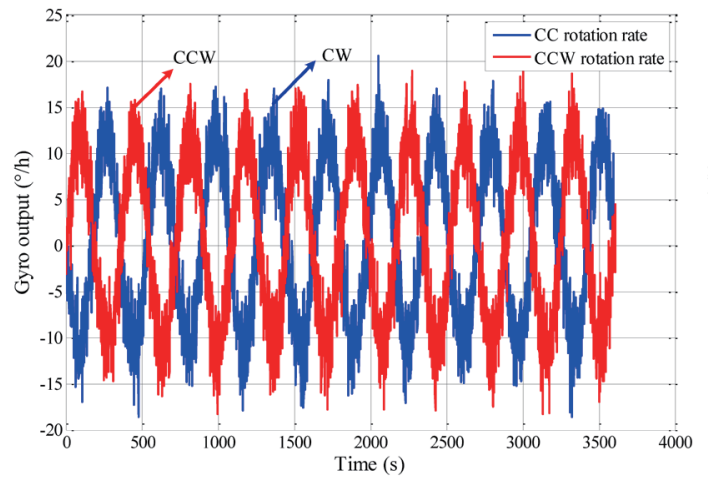

(a)

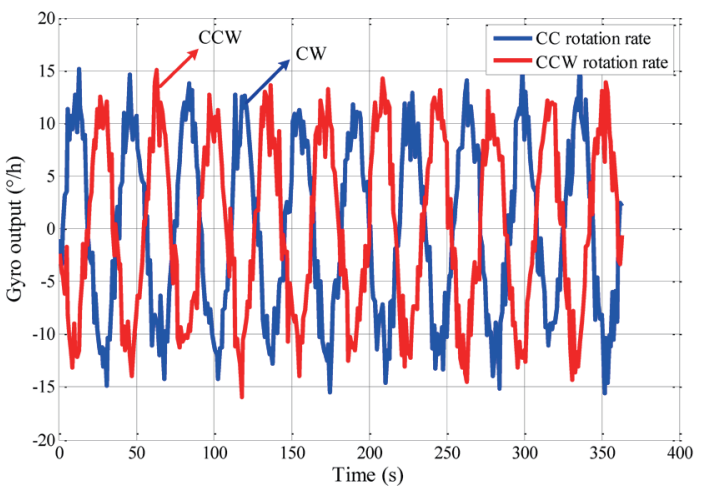

(b)

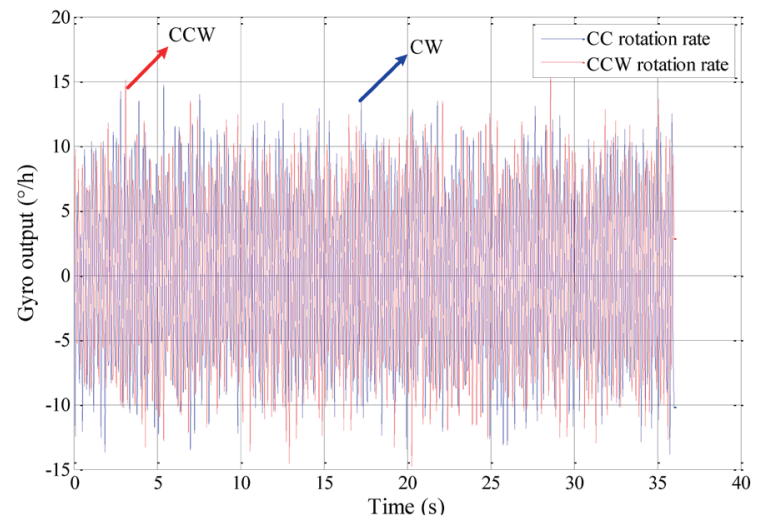

(c)

Fig. 8. (Color online) Gyro output when the turntable rotation rates are (a) $\pm 1 \%$, (b) $\pm 10 \%$, and (c) $\pm 100 \%$ s. 


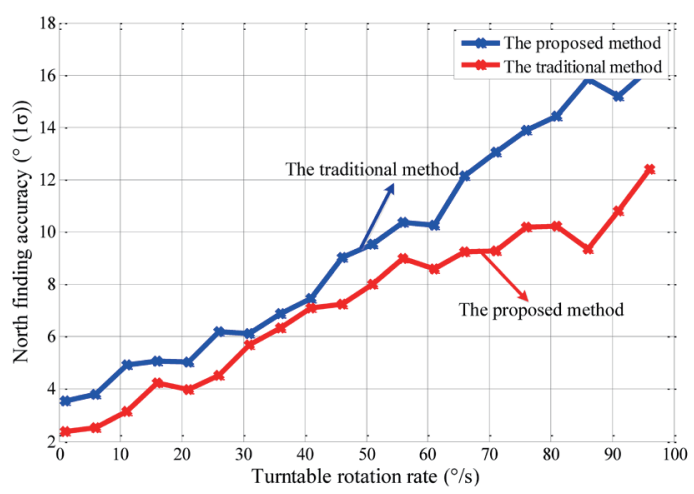

Fig. 9. (Color online) North finding angles obtained by proposed and traditional methods.

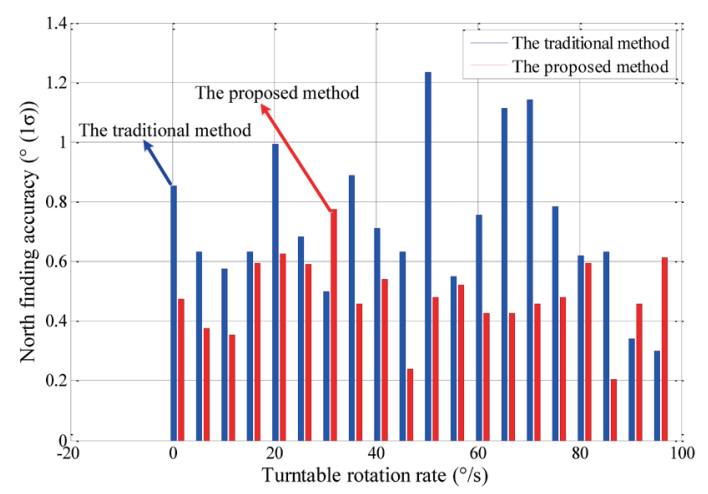

Fig. 10. (Color online) 1-sigma error bars of proposed and traditional methods.

As shown in Figs. 9 and 10, the north finding accuracy degrades with increasing turntable rotation rate. Thus, it is necessary to select the appropriate rotation rate carefully. The north finding accuracy is improved by the proposed method, which verifies the validity of this method. In order to compare the accuracy between the traditional method and the proposed method, the results at different rotation rates are shown in Table 2. As shown in Table 2, the north finding angle determined by the improved method is superior to that determined by the traditional method, and the stability of the MEMS gyro is improved by $32.6 \%$.

\subsection{Experimental results at different initial positions}

In order to further verify the performance of the proposed method, the initial direction of the gyro's sensitive axis was set to eight positions at $45^{\circ}$ intervals within a cycle, and the turntable rotation rate was set to $10 \% \mathrm{~s}$. Ten trials of the north finding experiments were carried out at each position, and the north finding results obtained by the traditional method and the proposed method based on the phase difference are shown in Table 3. From Table 3, it is obvious that the proposed method improved the north finding accuracy by $50 \%$ compared with the traditional method, which clearly proves the effectiveness of the method.

\subsection{Discussion}

In this study, a series of experiments were performed to validate the feasibility and effectiveness of the proposed method, and the experimental results are discussed in detail. The results prove the superior performance of the proposed method, which successfully improves the north finding accuracy. The main advantages of the proposed method are as follows.

(1) The north finding results are not affected by the turntable rotation rate instability error;

(2) The proposed bidirectional carouseling method is effective;

(3) Experiments under different conditions can be carried out to verify the theoretical deduction. 
Table 2

North finding angle of two methods based on different rotation rates.

\begin{tabular}{lcc}
\hline Method & Turntable rotation rate $(\%)$ & North finding angle $\left(^{\circ}\right)$ \\
\hline \multirow{3}{*}{ Traditional method } & 1 & 3.53 \\
& 10 & 4.91 \\
& 100 & 16.23 \\
\hline \multirow{3}{*}{ Proposed method } & \pm 1 & 2.38 \\
& \pm 10 & 3.16 \\
& \pm 100 & 12.41 \\
\hline
\end{tabular}

Table 3

North finding accuracy of two methods at different initial positions.

\begin{tabular}{ccc}
\hline Initial position $\left(^{\circ}\right)$ & Traditional method $\left[{ }^{\circ}(1 \sigma)\right]$ & Proposed method $\left[^{\circ}(1 \sigma)\right]$ \\
\hline 0 & 0.86 & 0.47 \\
45 & 0.90 & 0.51 \\
90 & 0.82 & 0.43 \\
135 & 0.87 & 0.49 \\
180 & 0.93 & 0.53 \\
225 & 0.89 & 0.48 \\
270 & 0.83 & 0.44 \\
315 & 0.88 & 0.50 \\
\hline
\end{tabular}

\section{Conclusions}

In order to improve the north finding accuracy, a bidirectional carouseling method based on the phase difference is proposed. With the low-pass filter removing the periodic term in the differential signal, the effect of gyro bias and random error on the north finding accuracy is effectively suppressed, and the turntable rotation rate instability error does not affect the north finding accuracy. The experimental results demonstrate that the proposed method can effectively achieve fast and high-precision MEMS gyro north finding. Our future work will be focused on reducing the effects of temperature and g-sensitivity to further improve the north finding accuracy.

\section{Acknowledgments}

This work was supported by the National Key Scientific Instrument and Equipment Development Project (2013YQ50791).

\section{References}

1 A. A. Trusov, I. P. Prikhodko, D. M. Rozelle, A. D. Meyer, and A. M. Shkel: Int. Conf. Solid-State Sens. (2013) 2531. https://doi.org/10.1109/Transducers.2013.6627321

2 Y. L. Wei and M. Lee: Proc. 2012 SICE Annu. Conf. (SICE, 2012) 2262. https://doi.org/10.1007/978-3-64223914-4 4

3 L. I. Iozan, M. Kirkko-Jaakkola, J. Collin, J. Takala, and C. Rusu: Meas. Sci. Technol. 23 (2012) 025005. https://doi.org/10.1088/0957-0233/23/2/025005

4 Y. J. Zhang, B. Zhou, M. L. Song, B. Hou, H. F. Xing, and R. Zhang: Sensors 17 (2017) 973. https://doi. org/10.3390/s17050973

5 H. F. Xing, B. Hou, and Z. H. Lin: Sensors 17 (2017) 2335. https://doi.org/10.3390/s17102335 
6 R. Arnaudov and Y. Angelov: Meas. Sci. Technol. 16 (2005) 2300. https://doi.org/10.1088/0957-0233/16/11/024

7 D. Lapadatu, B. Blixhavn, R. Holm, and T. Kvisteroy: IEEE/ION Position Location Navig. Symp. 1 (2010) 6. https://doi.org/10.1109/PLANS.2010.5507139

8 S. C. Wu, C. Liu, S. B. Yu, Z. Q. Wang, and J. R. Li: Int. Conf. MEC (IEEE, 2011) 994. https://doi.org/10.1109/ MEC.2011.6025632

9 I. P. Prikhodko, S. A. Zotov, and A. A. Trusov: Proc. 2011 16th Int. Solid-State Sensors, Actuators and Microsystems Conf. (IEEE, 2011) 2809. https://doi.org/10.1109/TRANSDUCERS.2011.5969216

10 B. M. Renkoski: M. S. thesis, Dept. Aeronaut. Astronaut., Massachusetts Inst. Technol. (2008).

11 B. R. Johnson, E. Cabuz, H. B. French, and R. Supino: IEEE/ION Position Location Navig. Symp. (2010) 168. https://doi.org/10.1109/PLANS.2010.5507133

12 A. A. Trusov, I. P. Prikhodko, S. A. Zotov, and A. M. Shkel: IEEE/ION IEEE Position Location Navig. Symp. (2012) 247. https://doi.org/10.1109/PLANS.2012.6236888

13 I. P. Prikhodko, S. A. Zotov, A. A. Trusov, and A. M. Shkel: J. Microelectromech. Syst. 22 (2013) 1257. https:// doi.org/10.1109/JMEMS.2013.2282936

14 I. P. Prikhodko, A. A. Trusov, and A. M. Shkel: IEEE Int. Conf. Micro Electro. Mech. Syst. (2012) 164. https:// doi.org/10.1109/MEMSYS.2012.6170119

15 Q. Wang, J, Xie, C. C. Yang, C. H. He, X. Y. Wang, and Z. Y. Wang: Appl.Opt. 54 (2015) 8944. https://doi. org/10.1364/ao.54.008944

16 R. Arnaudov and Y. Angelov: Meas. Sci. Technol. 16 (2005) 2300. https://doi.org/10.1088/0957-0233/16/11/024

17 N. Kolev, R. Arnaudov, and Y. Angelov: IEEE Technol. Appl. (2007) 464. https://doi.org/10.1109/ IDAACS.2005.283025

18 C. H. He, C. Yang, X. Wang, and Z. Wang: Appl. Opt. 52 (2013) 5303. https://doi.org/10.1364/AO.52.005303

19 J. Collin, M. Kirkko-Jaakkola, and J. Takala: IEEE Trans. Instrum. Meas. 64 (2013) 230. https://doi. org/10.1109/tim.2014.2335921

20 D. K. Upadhyay and T. Khanna: IEEE Int. Conf. Electron. Comput. Commun. Technol. (2015). https://doi. org/10.1109/CONECCT.2015.7383938

21 Analog Devices, Inc.: http://www.analog.com/cn/products/mems/inertial-measurement-units/adis16495.html

22 Butterworth Filter Design: http://www.electronics-tutorials.ws/filter/filter_8.html

\section{About the Authors}

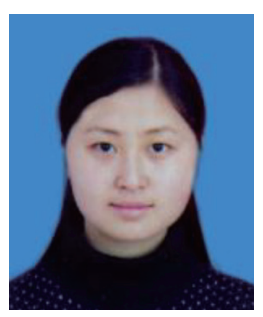

Xiaowen Cai received her Master's degree in Control Theory and Control Engineering from Xiangtan University of China, Xiangtan, China. She is currently pursuing her Ph.D. degree at Beihang University. Her research interests are related to inertial navigation and integrated navigation.

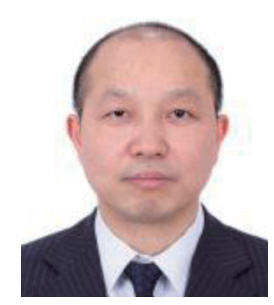

Chunxi Zhang received his Ph.D. degree in Optical Engineering from Zhejiang University. He has been a professor at Beihang University since 1999. His current research interests are in integrated navigation and optical fiber sensing technology.

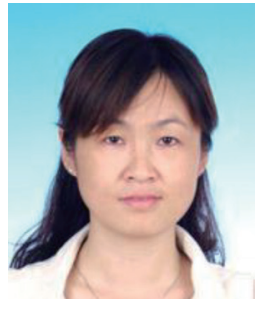

Shuang Gao received her Ph.D. degree in Optical Engineering from the School of Instrumentation Science and Opto-electronics Engineering (SIOE) at Beijing University of Aeronautics and Astronautics, Beijing, China, in 2008. Her research interests focus on inertial navigation and integrated navigation. 


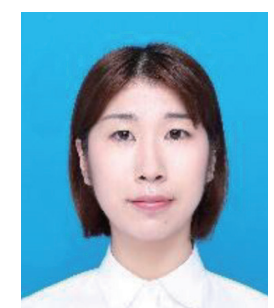

Ruoyu Zhang received her B.S. degree in Automation from China University of Petroleum. She is currently an M.S. candidate in Optical Engineering at Beijing University of Aeronautics and Astronautics. Her research interests focus on inertial navigation and integrated navigation. 